\title{
Familial aggregation of phenotypic expression of premature hair hy- popigmentation in the craniofacial region
}

\author{
Corey L. Black ${ }^{1}$ \\ ${ }^{1}$ University of Pittsburgh School of Dental Medicine, Pittsburgh PA, USA
}

\section{Abstract}

There are many patients who experience premature hypopigmentation of hair, but do not understand the underlying causes and potential dangers associated with them. The causes range from genetic predisposition to environmental influences such as tobacco use. Premature hypopigmentation of the hair shaft can also be associated with many syndromes; some which cause dental anomalies. Today, treatment options are limited for patients, although various studies are being done on mice to target the underlying mechanism of action. Understanding the differences between all of the possible causes of this particular phenotype can help clinicians better identify the symptoms, educate patients, and possibly modify treatment to suit the needs of each patient on an individual basis.

\section{Introduction}

Determination of the color of hair is a complicated process. There are genes that dictate color, texture, distribution, and number of stem cells [1]. Gray hair, also known as canities, is a phenotypic manifestation of a process in while melanocytes within hair follicles stop producing melanin. For unknown reasons, this process occurs with most individuals as the aging process proceeds. However, with a fair number of individuals, the process of pigmentation of the hair shaft begins much sooner than the average individual. Hair is said to gray prematurely when it occurs before the age of 20 in whites, 25 in Asians and 30 in Africans [1]. There have been many proposed causes of gray hair including stress, cigarette smoking, nutritional deficiencies, genetics, and a variety of syndromes. It is important for a clinician to understand the many possibilities of this phenotype in order to be able to identify patients who may display premature canities.

\section{Case Report}

\section{An 18-year-old African} American male, individual II.2 (Figure 1) began to experience canities prior to college admission in July of 2007. The patient noticed two gray hairs in the center of his scalp. Believing that they may have been caused by stress, he left them alone and started to use stress reduction
Citation: Black, C. (2015) Familial aggregation of phenotypic expression of premature hair hypopigmentation in the craniofacial region. Dentistry 3000. 1:a001 doi:10.5195/d3000.2015.31 Received: March 18, 2015

Accepted: March , 2015

Published: April 7, 2015

Copyright: (02015 Black. This is an open access article licensed under a Creative Commons Attribution Work 4.0 United States License. Email: clb196@pitt.edu techniques such as exercise and constant breaks when studying. He also started to take a multi-vitamin to ensure that the presentation of the gray hairs was not due to a B-12 or iron deficiency. However, as the years progressed, the number of gray hairs continued to increase at a rate of approximately $10-15$ hairs per year. At the age of 22, the phenotypic expression of the gray hairs extended to his facial hair, including a few hairs along the jaw line and a significant amount extending across the anterior region of the neck. Within a span of 8 years, the number of hypopigmented hairs increased from 2 to approximately 80 (Figure 2). 
The patient's medical history was negative for any major systemic disease. There were no medications, other than antibiotics and mentation appeared only in the anterior neck region and began at the age of 25 (Figure 2). This may be explained by variable expressivity

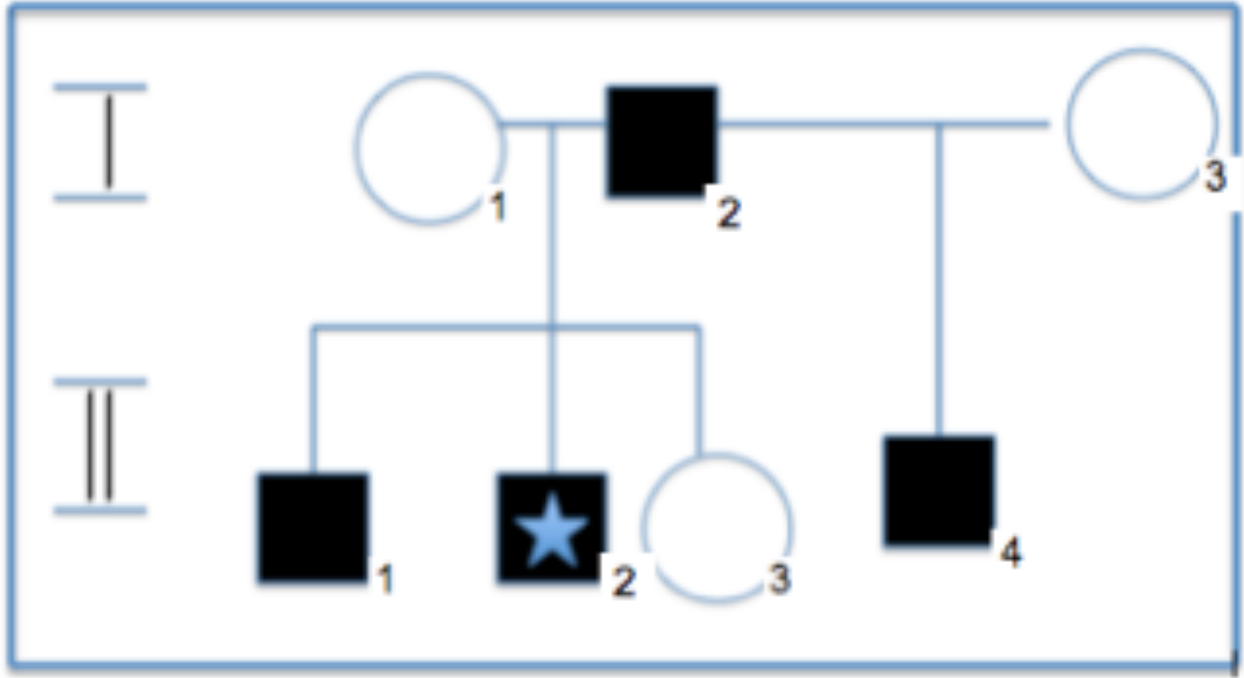

Figure 1. A pedigree shows that the males in generations II and I are affected by premature hypopigmentation. Individual II.4 is also affected, regardless of the fact that his maternal genome differs from the other progeny in the second generation. The individual of the case report, II.2 is demarcated with a "star".

multivitamins. He annually visits a primary care physician and had no major hospitalizations. The patient has a family history of cancer, hypertension, and diabetes on the maternal side. There is a history of heart and liver disease on the paternal side.

When examining the family history of the patient, it appears that the male progeny within the immediate generation are affected by hypopigmentation of the hair follicles of the craniofacial area. After consulting with his father, it was revealed that the father of the patient was affected by the same phenotype of premature hypopigmentation at the age of 20 . There was a similar manifestation of the initial hypopigmentation appearing in the scalp and subsequent hypopigmentation in the anterior region of the neck.

The individual II.1, a direct male descendant of I.1, also experienced a similar phonotypical manifestation. However, the hypopig- when compared to the early presentation of this phenotype at age 18 in individual II.2. Individual II.1 also experienced male pattern baldness at the age of 22 . Therefore, it is unclear whether the follicles on the scalp were affected by hypopigmentation. Individual I.1 also has anoth-

which is considered premature. The patient's sister, individual II.3 does not express any symptoms of hypopigmentation (Figure 1).

\section{Discussion}

Today, the exact mechanism of canities is unknown. However, when considering the underlying cause of premature hypopigmentation, one must consider multiple mechanisms, encompassing environmental, systemic, and genetic factors. Also, the expressivity of the phenotype varies for each individual. In this case, there seems to be a strong genetic link, originating from the paternal side of the family.

From a genetic standpoint, premature graying of hair occurs more commonly without any underlying pathology but it is thought to be inherited in autosomal dominant pattern [2]. It appears that the genotype is one that includes multiple genes and peptides that affect the enzymes responsible for melanin production.

The activity of the tyrosinase enzyme is thought to be crucial in this process. One study observed

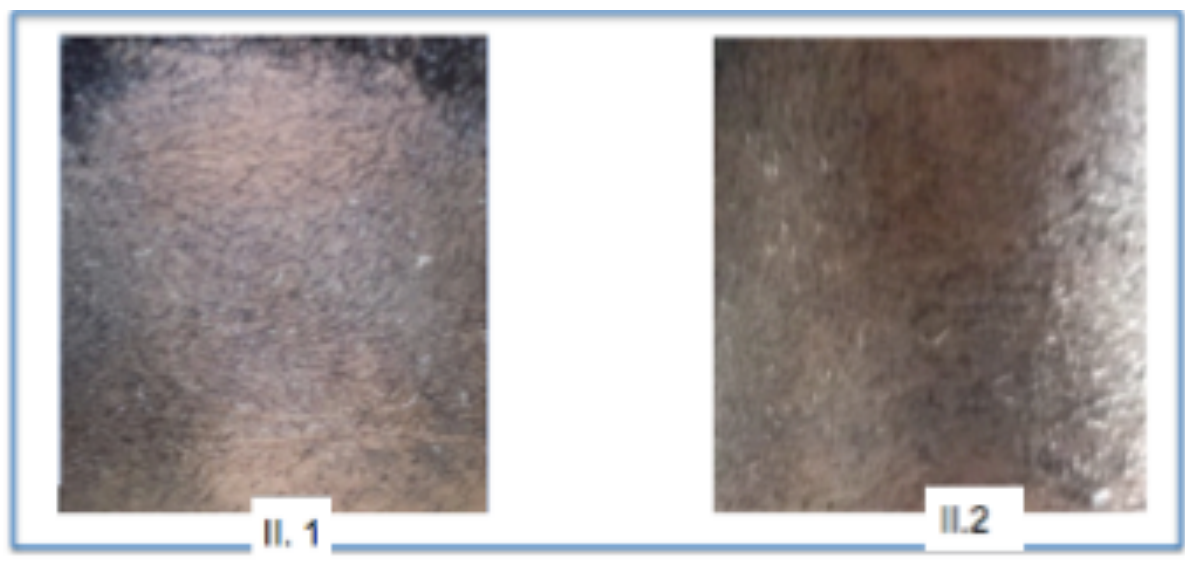

Figure 2. The photo on the left is individual II.1 shown in the pedigree. The photo on the right is individual II.2.

er son from a previous marriage who is also affected by hypopigmentation. It is unsure at what age the phenotype was expressed; however it was before the age of 30 , the melanin in albino-mouse hair follicles. In this study, a point mutation in the tyrosinase gene was corrected in some of the murine follicles with RNA-DNA chimeric oligo- 
nucleotides. A recombinant retrovirus (pLmelSN) from the locus of Streptomyces Antibioticus genome was used to induce melanin production in vitro [3]. These results indicate that the tyrosinase inactivity may be a possible link to hair hypopigmentation and some factors may cause the premature inactivation of these enzymes.

Because the hair cycle and growth process are complicated, it makes it difficult to study specific aspects of the cycle - especially melanin production. However, other genes are thought to be involved in this very complex process. It is suspected that mice deficient in $\mathrm{Bcl}$ 2 and $B c l-x$ genes are normal at birth but develop gray hair after a few weeks [2]. Also, a germ line mutation in Syntaxin 17 gene in horses causes premature gray hair in the homozygous genotype [4]. Based on the patient's medical and family history, there seems to be a strong genetic link to the cause of this particular phenotype.

There are many diseases, deficiencies, and syndromes that have been linked to premature hypopigmentation of hair. Some of these include Vitiligo, Werner's syndrome, thyroid disease, vitamin B12, iron, and calcium deficiencies. One syndrome, known as Book's syndrome, contains hypodontia and premature graying as phenotypic traits [2]. Clinicians should be aware of this syndrome and its possible physical manifestations. The patient has no known syndromes or deficiencies. Therefore, it is unlikely that this is the cause of the phenotype.

The environment can also play a crucial role in early hypopigmentation of hair. Studies suggest that there is a significant association between tobacco use and aging on graying of hair [5]. Another study suggests that prema- ture graying of the hair is associated with premature cardiovascular disease. It should probably be regarded as a coronary risk factor and used to identify patients at increased risk [5]. These environmental influences should be explained to patients who may not contain such relevant knowledge. Since cardiovascular disease is present in the family medical history, it should be explained to the patient that there may be a possible link between premature canities and heart disease. Hence, future testing should be done.

\section{Conclusion}

Clinicians must be able to recognize premature hypopigmentation of hair and understand the many causes of it. The ultimate goal is to know that the hypopigmentation phenotype can be explained by a number of factors. In order to understand the exact cause, clinicians must ask the appropriate questions, comprehending that the physical appearance of a few premature gray hairs can provide information about unknown underlying factors. Once the real cause is known, treatment may be modified to ensure that the patient receives the best quality of care when under the care of that particular clinician.

\section{References}

1. Epidemiological and investigative study of premature graying of hair in higher secondary and preuniversity school children. Bhat RM, Sharma R, Pinto AC, Dandekeri S, Martis J. Int J Trichology. 2013 Jan;5(1):17-21. doi: 10.4103/09747753.114706. PMID:23960391

2. Tobin, D. \& Trueb, R. (2010). Natural Hair Graying. In Aging Hair (pp.
208-210). New York, New York: Springer Science \& Business Media.

3. The hair follicle and its stem cells as drug delivery targets. Hoffman RM. Expert Opin Drug Deliv. 2006 May;3(3):437-43. Review. PMID:16640502

4. Polymorphisms in the syntaxin 17 gene are not associated with human cutaneous malignant melanoma. Zhao ZZ, Duffy DL, Thomas SA, Martin NG, Hayward NK, Montgomery GW. Melanoma Res. 2009 Apr;19(2):80-6. doi: 10.1097/CMR.0b013e328322fc45. PMID:19209086

5. Premature hair graying: a probable coronary risk factor. Gould L, Reddy CV, Oh KC, Kim SG, Becker W. Angiology. 1978 Nov;29(11):800-3. PMID:727561 